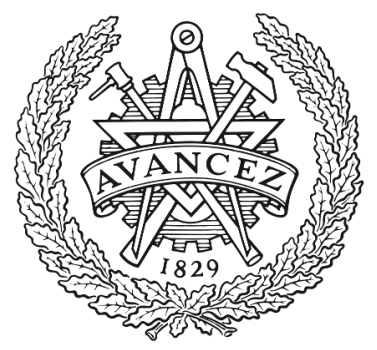

CHALMERS

UNIVERSITY OF TECHNOLOGY

\title{
A pluralist approach to the formalisation of mathematics
}

Downloaded from: https://research.chalmers.se, 2023-04-26 10:29 UTC

Citation for the original published paper (version of record):

Adams, R., Luo, Z. (2011). A pluralist approach to the formalisation of mathematics. Mathematical

Structures in Computer Science, 21(4): 913-942. http://dx.doi.org/10.1017/S0960129511000156

N.B. When citing this work, cite the original published paper. 


\section{A pluralist approach to the formalisation of}

Article in Mathematical Structures in Computer Science · August 2011

DOI: 10.1017/50960129511000156 · Source: DBLP

2 authors:

Dobin Adams

Chalmers University of Technology

13 PUBLICATIONS 82 CITATIONS

haohui Luo

Royal Holloway, University of London

83 PUBLICATIONS $\mathbf{1 , 4 9 6}$ CITATIONS

SEE PROFILE

SEE PROFILE

Some of the authors of this publication are also working on these related projects:

Proof Assistants for Natural Language Semantics View project

Reverse Mathematics in Dependent Type Theory View project 


\title{
A Pluralist Approach to the Formalisation of Mathematics
}

\author{
Robin Adams and Zhaohui Luo \\ Department of Computer Science \\ Royal Holloway, University of London \\ Egham, Surrey TW20 OEX, U.K. \\ Email: $\{$ robin, zhaohui\}@cs.rhul.ac.uk
}

Received 15 December 2010

\begin{abstract}
We present a programme of research for pluralist formalisations - formalisations that involve proving results in more than one foundation.

A foundation consists of two parts: a logical part that provides a notion of inference, and a non-logical part that provides the entities to be reasoned about. A logic-enriched type theory (LTT) is a formal system composed of such two separate parts. We show how LTTs may be used as the basis for a pluralist formalisation.

We show how different foundations may be formalised as LTTs, and also describe a new method for proof reuse. If we know that a translation $\Phi$ exists between logic-enriched type theories (LTTs) $S$ and $T$, and we have formalised a proof of a theorem $\alpha$ in $S$, we may wish to make use of the fact that $\Phi(\alpha)$ is a theorem of $T$. We show how this is sometimes possible by writing a proof script $M_{\Phi}$. For any proof script $M_{\alpha}$ that proves a theorem $\alpha$ in $S$, if we change $M_{\alpha}$ so it first imports $M_{\Phi}$, then the resulting proof script will still parse, and will be a proof of $\Phi(\alpha)$ in $T$.

In this paper, we focus on the logical part of an LTT-framework and show how the above method of proof reuse is done for four cases of $\Phi$ : inclusion, the double negation translation, the $A$-translation, and the Russell-Prawitz modality. This work has been carried out using the proof assistant Plastic.
\end{abstract}

\section{Introduction}

When formalising a piece of mathematics, we must first choose a foundation - a formal language in which the mathematical entities can be defined, and theorems and proofs about these entities can be written. Usually, such a foundation consists of two parts: a non-logical part for defining the mathematical entities to be reasoned about, and a logical part that formalises the underlying logical inference (e.g., a system of logic with a set of axioms and rules of deduction that determines which proofs are valid)

Much mathematical work involves working with more than one foundation: comparing the theorems that are provable in each, defining translations between foundations, comparing the class of models of each foundation, etc.

For example, work in set theory often involves comparing several different set theories 
and the theorems that can be proven in each. Similar work compares the theorems provable in different fragments of first-order arithmetic (Hájek and Pudlák, 1998). The large research project known as Reverse Mathematics uses several systems of second-order arithmetic (Simpson, 1999), and similar work has been done in higher-order arithmetic (Kohlenbach, 2005).

In this paper, we propose a programme of research for conducting pluralist formalisations - formalisations of pieces of mathematics that involve more than one foundation.

The prevailing paradigm in the formalisation of mathematics so far has been to choose one foundation, implement a proof assistant that constructs formal proofs in that foundation, and proceed to build up a large library of formalised results in that foundation. Some proof assistants offer the choice of a small number of different foundations - for example, LEGO (Pollack, 1994) implements four, and Coq (Coq, 2004) offers the user the choice of whether Set should be predicative or impredicative.

There are also proof assistants that implement logical frameworks, such as Isabelle (Paulson, 1994) and the Edinburgh LF (Harper et al., 1987; Harper et al., 1993) as implemented in Twelf (Pfenning and Schürmann, 1999). These allow more than one foundation to be represented, and often provide support for representing and reasoning about relations and translations between foundations.

If we wish to formalise a large piece of mathematics that involves proving results in several different foundations, it will be essential that we can reuse proofs carried out in one foundation within another. We shall therefore investigate the following questions: What must a logical framework provide in order to be suitable for a pluralist formalisation? How should we represent the different foundations within this logical framework? And how can we reuse a proof script written in one foundation when working in another?

Our answer to the second question is that the foundations should be represented as logic-enriched type theories (LTTs). We shall argue that LTTs possess some advantages over other systems of logic for the purposes of a pluralist formalisation. Our method of proof reuse relies on the two systems in question being declared in a fairly similar way. Thus, when choosing a family of systems with which to conduct a pluralist formalisation, we require one that will allow for a uniform presentation and treatment of a large number of different foundations. LTTs provide such a uniform framework.

We shall present a logical framework suitable for representing LTTs, discuss several issues in the construction of LTTs, and present a method for proof reuse between LTTs. The method of proof reuse that we present is quite general: we shall show with several quite varied examples how, given a translation from one foundation $S$ to another foundation $T$, we are able to take proof scripts in $S$ and reuse them when working in $T$.

We work with LTTs in this paper, but our method is also usable with type theories, systems of first-order logic, etc. It should therefore be useful to people working in many different areas of the formalisation of mathematics.

It is important to note that the LTT-approach to formalisation involves formalisation of the non-logical entities as well as that of the underlying logic. Traditionally, the studies of a logical framework such as Edinburgh LF or the system Twelf have mainly focused on the formal representations of logical systems and usually pay less attention to the non-logical parts of a mathematical system. The LTT-approach is different: it takes the 
formalisation of the non-logical entities seriously and this is also a key part of the pluralist approach to formalisation and proof reuse. We shall discuss this issue, although the focus of the current paper is mainly on the logical part of the LTT-approach.

There has not been much work on pluralist formalisations in the literature, but there has been quite a lot of research into the related problem of sharing results produced using different proof assistants. We discuss this work in Section 6.1.

\subsection{Outline}

In Section 2, we shall discuss some general issues around the formalisation of mathematics using more than one foundation. The type-theoretic framework of LTTs is introduced in Section 3. In Section 4, we shall describe our method for proof reuse in more detail and, in Section 5, the method is applied to four examples and it is shown how these have been formalised using the proof assistant Plastic.

\section{A Pluralist Approach to the Formalisation of Mathematics}

\subsection{Mathematics with Different Foundations}

When building a foundational system for mathematics, one faces various choices. For example, two of the decisions that must be made are: whether the logic shall be classical or intuitionistic; and whether impredicative definitions are allowed, or only predicative.

Each of the four possible combinations of these options has been advocated as a foundation for mathematics at some point in history.

- Impredicative classical mathematics. This is arguably the way in which the vast majority of practising mathematicians work. Zermelo-Fraenkel Set Theory (ZF) is one such foundation. The proof checker Mizar (Muzalewski, 1993) has been used to formalise a very large body of impredicative classical mathematics. The foundation HOL, as implemented in the proof assistants Isabelle (Nipkow et al., 2002) and HOLLight (Harrison, 1996), is another.

- Impredicative constructive mathematics. Impredicative types theories such as ECC/UTT (Luo, 1994) and CIC (Bertot and Castéran, 2004) are examples of such foundations. These have been implemented by the proof checkers LEGO (Luo and Pollack, 1992) and Coq (Coq, 2004). There are also impredicative constructive set theories, such as Intuitionistic Zermelo-Fraenkel (IZF).

- Predicative classical mathematics. This was the approach taken by Weyl in his influential monograph of 1918, Das Kontinuum (Weyl, 1918). Stronger predicative classical systems have been investigated by Feferman (Feferman, 2005) and Schütte (Schütte, 1965).

- Predicative constructive mathematics. Its foundations are provided, for example, by Martin-Löf's type theory (Nordström et al., 1990; Martin-Löf, 1984), whose variants are implemented in the proof assistants Agda (Agda, 2008) and NuPRL (Constable et al., 1986). There are also predicative constructive set theories, such as Constructive Zermelo-Fraenkel set theory (CZF). 
The two choices listed above are by no means the only ones that must be considered when designing a foundation. We must also consider whether equality should be intensional or extensional; which choice principles should be allowed; etc. A wide variety of mathematical foundations are in use today.

One foundation may sometimes be an extension of another. For example, ZF is an extension of IZF; that is, everything provable in IZF is provable in ZF. There can also be translations between these system, such as the double negation translation (Gödel, 1933) from the classical system Peano Arithmetic to the intuitionistic Heyting Arithmetic.

When beginning a pluralist formalisation, we must consider how the several different formalisations involved can be captured by a family of formal systems, in a manner that is uniform enough for proof reuse to be practicable. As we have argued elsewhere (Adams and Luo, 2010), logic-enriched type theories are able to capture a remarkably wide range of foundations very faithfully, in a very uniform manner.

\subsection{Proof Reuse in Logic-Enriched Type Theories}

In this paper, we shall present a method for proof reuse. Suppose we have two foundations $S$ and $T$, and a translation from $S$ to $T$. When we are working in $T$, we want to be able to reuse proof scripts formalising results in $S$.

Further, we shall be greedy. We do not want to prove a lemma relating $S$ and $T$, and then have to apply that lemma many times. We do not want to write a program that will automatically translate an $S$-proof script into a $T$-proof script. We want to be able to take an $S$-proof script and reuse it - immediately, without any modification - as a $T$-proof script.

There are two particular situations that we wish to consider:

1 We have two foundations $S$ and $T$, and $S$ is a subsystem of $T$. If we have shown that $S \vdash \alpha$, then we can immediately make use of the fact that $T \vdash \alpha$. When formalising a piece of mathematics that makes use of this sort of step, we wish to take a proof script that formalises a proof of $\alpha$ in $S$, and use this script to provide us with a proof of $\alpha$ when working in $T$.

2 More generally, we have two foundations $S$ and $T$, and a translation $\Phi: S \rightarrow T$; that is, a mapping from the language of $S$ to the language of $T$ such that, if $S \vdash \alpha$, then $T \vdash \Phi(\alpha)$. When formalising a piece of mathematics that makes use of this sort of step, we wish to take a proof script that formalises a proof of $\alpha$ in $S$, and use this script to provide us with a proof of $\Phi(\alpha)$ when working in $T$.

Case 1 is a special case of case 2, where the translation $\Phi$ is the inclusion from $S$ to $T$.

We shall be working in this paper with logic-enriched type theories (LTTs). These are systems of logic that consist of a type theory, that defines the mathematical objects we will be dealing with, and a separate logical component, for stating and proving propositions about those objects.

In case 1 above, we can sometimes arrange it so that $\mathrm{LTT}_{S}$, which represents $S$, is a subsystem of the $\mathrm{LTT}_{T}$, which represents $T$. Suppose we have proof scripts $M_{S}$ and $M_{T}$ that define $\mathrm{LTT}_{S}$ and $\mathrm{LTT}_{T}$, respectively. If a proof script imports $M_{S}$ and proves 


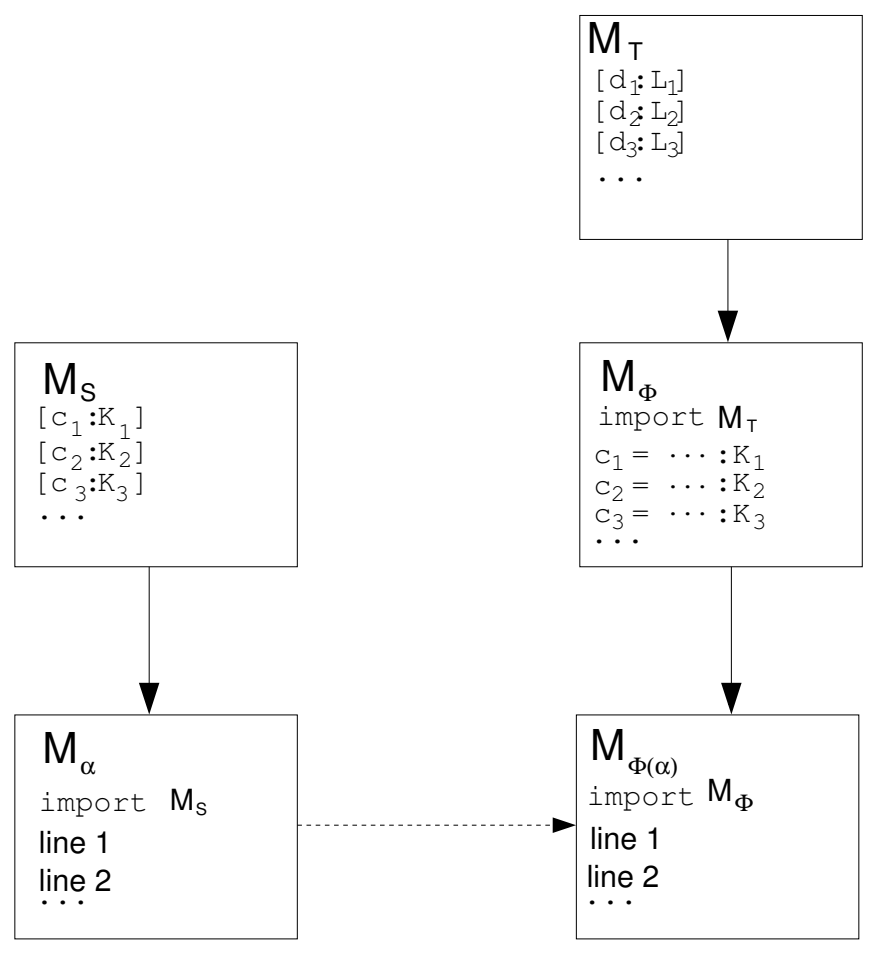

Fig. 1. Proof Reuse Between Two LTTs

a theorem $\alpha$, the proof script will still parse if we change it to import $M_{T}$ instead. This idea was made use of in (Adams and Luo, 2010), where we proved several results in the predicative $\mathrm{LTT}_{W}$, and were able to immediately reuse those proof scripts in an impredicative LTT that extends $\mathrm{LTT}_{W}$.

However, such an approach is quite fragile; it depends on us using the same names for constants in $M_{S}$ and $M_{T}$. It is not certain that we can always define $\mathrm{LTT}_{S}$ and $\mathrm{LTT}_{T}$ in such a way that $\mathrm{LTT}_{S}$ is a subsystem of $\mathrm{LTT}_{T}$. Moreover, this approach cannot handle the more general case 2 above.

The approach we present in this paper is as follows. Suppose we have a translation $\Phi$ from $\mathrm{LTT}_{S}$ to $\mathrm{LTT}_{T}$. Given proof scripts $M_{S}$ and $M_{T}$ that define $\mathrm{LTT}_{S}$ and $\mathrm{LTT}_{T}$, we shall construct a proof script $M_{\Phi}$ that imports $M_{T}$, and then defines every constant that was declared in $M_{S}$ (see Fig. 1).

A proof script $M_{\alpha}$ that imports $M_{S}$ will still parse if we change it to import $M_{\Phi}$ instead. Further, we can write $M_{\Phi}$ in such a way that, if $M_{\alpha}$ provides a proof of $\alpha$ under $M_{S}$, then it provides a proof of $\Phi(\alpha)$ under $M_{\Phi}$.

We shall show in this paper how this can be done when $\mathrm{LTT}_{S}$ is a subsystem of $\mathrm{LTT}_{T}$, and in three other cases: the double-negation translation from classical to intuitionistic logic; the $A$-translation from intuitionistic logic to itself; and the Russell-Prawitz modality from first-order logic (classical or intuitionistic) to second-order logic. 


\section{A Type-Theoretic Framework for Pluralist Formalisations}

Our approach to pluralist formalisations is based on a uniform framework in which mathematics with different foundations can be formalised. For this purpose, the type-theoretic framework of Logic-enriched Type Theories (LTTs) is particularly appropriate.

Logic-enriched type theories were first studied by Aczel and Gambino to investigate type-theoretic interpretations of constructive set theory (Aczel and Gambino, 2002; Gambino and Aczel, 2006). An LTT is a formal system consisting of a type-theoretic component that provides types and terms; and a logical component that provides propositions and proofs. The intention is that the types and terms describe the collection of mathematical objects we are concerned with, and the logical component to reason about those objects.

We shall present three logical frameworks in this section: LF, a Church-typed version of Martin-Löf's Logical Framework ${ }^{\dagger}$, and its two extensions $\mathrm{LF}^{\prime}$, intended for representing an LTT, and LF $_{\text {LTT }}$ intended for representing several LTTs simultaneously.

The type-theoretic framework is a method of specifying LTTs within a logical framework. The LTTs specifiable this way are capable of expressing a wide spectrum of foundations for mathematics in a uniform way. It was first proposed in (Luo, 2006) $)^{\ddagger}$. In (Adams and Luo, 2007; Adams and Luo, 2010), the authors studied one of the systems in this framework - a logic-enriched type theory $\mathrm{LTT}_{W}$ that gives a modern type-theoretic version of Weyl's system for predicative mathematics — and used $\operatorname{LTT}_{W}$ to formalise Weyl's predicative mathematics (Weyl, 1918) in the proof assistant Plastic.

In this section, we shall review the logical framework LF and its extension $\mathrm{LF}^{\prime}$. We then introduce its extension $\mathrm{LF}_{\mathrm{LTT}}$, and describe how $\mathrm{LF}_{\mathrm{LTT}}$ may be used to specify LTTs. We then present the type-theoretic framework, and give examples of logic-enriched type theories that can be specified in the framework.

\subsection{The Logical Framework LF}

A logical framework, such as Martin-Löf's logical framework (Nordström et al., 1990) and its Church-typed version LF (Luo, 1994), is a dependent type system, together with a method for representing other formal systems within that type system.

Here, we introduce LF briefly and fix our notations. The full details of LF, including its rules, can be found in Chapter 9 of (Luo, 1994).

3.1.1. Basic Constructions The system LF deals with kinds, and objects. The kinds are:

- Type - the kind of types;

- $\operatorname{El}(A)$ — the kind of objects of type $A$; and

$\dagger$ The framework LF should not be confused with the Edinburgh Logical Framework (Harper et al., 1987; Harper et al., 1993), which is also called LF. (It is unfortunate that the same name is used for both.) One of the main differences between LF and the Edinburgh LF is that the former system is intended to be used to specify type theories, and hence allows computation rules to be declared.

$\ddagger \mathrm{LF}$ has a variant called $\mathrm{PAL}^{+}$(Luo, 2003), where applications are fully applied or saturated. In (Luo, 2006), we adopted the notations of $\mathrm{PAL}^{+}$; but this is inessential and we shall use LF and the associated notations in this paper. 
The formation rule for $N$

$$
N \text { : Type }
$$

The introduction rules for $N$ (constructors)

$$
\begin{array}{rll}
0 & : & N \\
\text { succ } & : & (N) N
\end{array}
$$

The elimination rule over types for $N$

$$
\begin{aligned}
\mathcal{E}_{T}^{N}: \quad & (C:(N) \text { Type }) \\
& (c: C(0))(f:(n: N)(x: C(n)) C(\operatorname{succ}(n))) \\
& (z: N) C(z)
\end{aligned}
$$

The computation rules for $N$

$$
\begin{aligned}
\mathcal{E}_{T}^{N}(C, c, f, 0) & =c: C(0) \\
\mathcal{E}_{T}^{N}(C, c, f, \operatorname{succ}(n)) & =f\left(n, \mathcal{E}_{T}^{N}(C, c, f, n)\right): C(\operatorname{succ}(n))
\end{aligned}
$$

Fig. 2. The type of natural numbers.

- $(x: K) K^{\prime}$ - the kind of dependent functional operations $f$ which can be applied to any object $k$ of kind $K$ to form the application $f k$ of kind $[k / x] K^{\prime}$.

We often omit $E l$ and write $E l(A)$ simply as $A$. We write $K \rightarrow K^{\prime}$ for $(x: K) K^{\prime}$ when $x$ does not occur free in $K^{\prime}$.

When writing objects in the form of application, we shall sometimes write $f\left(a_{1}, \ldots, a_{n}\right)$ for $f a_{1} \ldots a_{n}$, and often use the infix-form of binary operators: for instance, we write $A \times B$ and $P \wedge Q$ for $\times(A, B)$ and $\wedge(P, Q)$, respectively.

Two objects are definitionally equal in LF if they are $\beta \eta$-convertible.

The system LF is intended for specifying type theories that deal with types and terms. The intention is that:

— the types are represented by the objects of kind Type;

- the terms of type $A$ are represented by the objects of kind $\operatorname{El}(A)$;

— the objects of kind $(x: K) K^{\prime}$ represent meta-functions on the type theory's syntax.

3.1.2. Specification of Type Theories A type theory is specified in LF by declaring constants, each with a kind; and computation rules. These declarations have the effect of extending LF with additional rules (see (Luo, 1994) for the details).

Typically, a type in a type theory comes with its rules of formation, introduction, elimination and computation. We represent this type in LF by declaring constants corresponding to the formation, introduction and elimination rules; and declaring equality rules corresponding to the computation rules.

For example, the type $N$ of natural numbers can be specified as in Figure 2 where, for 
instance, the following introduction rule

$$
\frac{\Gamma \vdash n: N}{\Gamma \vdash \operatorname{succ}(n): N}
$$

is specified by means of the declaration of the constant succ.

By this method, LF can specify type theories that contain inductive and co-inductive types, predicative and impredicative universes, inductive-recursive types, and others.

\subsection{Logic-enriched Type Theories}

The system LF is a suitable language for specifying type theories which deal with just types and terms. There is a single kind Type of all the types in the type theory.

If we wish to use a system to state and prove mathematical theorems, we must have some way of introducing logical propositions. In a type theory, one may do this by identifying propositions with types (e.g., in Martin-Löf's type theory, every proposition is a type and vice versa) or by taking propositions as types, but not vice versa (e.g., in ECC/UTT (Luo, 1994), every proposition is a type, but not every type is a proposition).

However, if one takes the view that logical propositions and data types should be completely separate, then one will wish to work in a different kind of system; and LF will not be adequate for specifying this different kind of system.

Logic-enriched type theories (LTTs) (Gambino and Aczel, 2006) are formal systems where there is a complete separation between (logical) propositions and (data) types. The syntax of an LTT consists of four categories of expression: types, terms, propositions, and proofs (or derivations).

An LTT thus naturally falls into two components, or 'worlds': the type-theoretic component, and the logical component. This allows for a lot of flexibility in the design of an LTT, as we can change one component without affecting the other (e.g. we can add excluded middle to the logical component, without changing the type-theoretic component). This makes LTTs suitable for capturing many different mathematical foundations.

The two components do, however, interact. The logical world may depend on the type-theoretic world: for example, given an inductive type such as $N$ or $\operatorname{List}(A)$, we may choose to introduce a rule of deduction allowing propositions to be proved by induction.

In order to specify an LTT adequately, an extension of LF is called for. This extended logical framework is obtained by extending LF by adding a new kind Prop, that stands for the world of logical propositions, and a new kind constructor Prf:

$$
\frac{\Gamma \text { valid }}{\Gamma \vdash \text { Prop kind }} \quad \frac{\Gamma \vdash P \text { : Prop }}{\Gamma \vdash \operatorname{Prf}(P) \text { kind }}
$$

This extended framework was first proposed in (Luo, 2006) and further studied in (Adams and Luo, 2010) and, in the latter paper, we have called it $L F^{\prime}$.

The intention is that:

— the types are represented by the objects of kind Type;

- the terms of type $A$ are represented by the objects of kind $\mathrm{El}(A)$;

- the propositions are represented by the objects of kind Prop; 
Conjunction

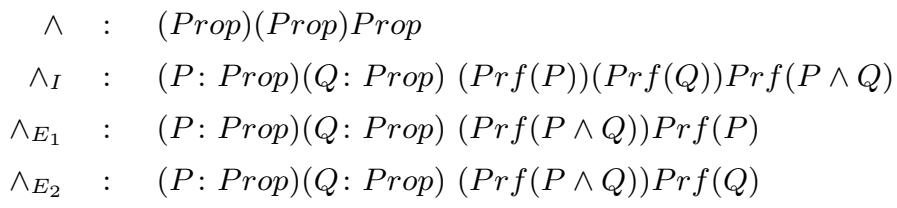

Universal quantifier

$$
\begin{array}{rll}
\forall: & (\text { A: Type })(P:(A) \text { Prop }) \text { Prop } \\
\forall_{I}:: & (\text { A: Type })(P:(A) \operatorname{Prop})((x: A) \operatorname{Prf}(P(x))) \operatorname{Prf}(\forall(A, P)) \\
\forall_{E}: \quad(A: \text { Type })(P:(A) \operatorname{Prop})(\operatorname{Pr} f(\forall(A, P)))(a: A) \operatorname{Pr} f(\operatorname{P}(a))
\end{array}
$$

Negation

$$
\begin{array}{rll}
\neg & : & (\text { Prop }) \text { Prop } \\
D N & : & (P: \text { Prop })(\operatorname{Prf}(\neg \neg P)) \operatorname{Prf}(P) \\
\ldots & \ldots & \ldots
\end{array}
$$

Fig. 3. Logical operators and direct proofs in the classical FOL.

— the proofs of a proposition $P$ are represented by the objects of kind $\operatorname{Prf}(P)$.

An LTT is specified in $L F^{\prime}$ by declaring constants and computation rules. Each declaration has the effect of extending $L F^{\prime}$ with new rules; see (Adams and Luo, 2010).

3.2.1. Logics. The logic in an LTT is specified by declaring constants for the logical operators and the associated rules.

For example, say we wish an LTT's logical component to consist of classical first-order logic in their logical part. This can be introduced by declaring the constants that stand for the logical operators, and constants that stand for the associated inference rules. The logical operators $\wedge, \forall$ and $\neg$ and some of their associated rules of inference are specified in Figure 3. Other logical operators can be introduced in a similar way.

\subsubsection{Remarks}

1 The quantifier $\forall$ declared here can only be used to quantify over a type; that is, for a formula $\forall(A, P)$, or $\forall x: A, P(x)$ in the usual notation, $A$ must be a type.

In particular, since Prop is not a type (it is a kind), one cannot form a proposition by quantifying over Prop. Higher-order logical quantification such as $\forall X: P r o p$. $X$, as found in impredicative type theories such as System F (Girard, 1986) and the Calculus of Constructions (Coquand and Huet, 1988), is not possible with this constant.

Similarly, since propositions are not types $(\operatorname{Pr} f(P)$ is a kind, not a type), one cannot quantify over the proofs of a proposition, either.

When designing an LTT, we can thus choose whether to allow first- or higher-order 
quantification. Contrast this situation with a type theory such as ECC or UTT (Luo, 1994), where it would not be possible to restrict quantification to the datatypes, since Prop is a type and every proposition is a type.

2 An LTT is specified in the framework, not just by specifying a collection of constants, but also by specifying computation rules. Computation rules are needed in the typetheoretic component for specifying inductive data types, universes, etc. Computation rules in the logical component are also sometimes needed; they were used, for example, in (Adams and Luo, 2010) in the specification of typed sets.

The ability to specify computation rules is the most important difference between the Martin-Löf family of logical frameworks, including $L F$ and $L F^{\prime}$, and the Edinburgh LF (Harper et al., 1993).

3 If we have introduced a universe that contains the empty type and the type of natural numbers, we can then prove, internally in the type-theoretic framework, that Peano's fourth axiom for natural numbers holds (i.e., the proposition $\forall x: N .\left(s[x] \neq_{N} 0\right)$ holds). This is similar to Martin-Löf's type theory, where without the presence of a type universe, one cannot prove Peano's fourth axiom internally (Smith, 1988).

\subsection{The Type-Theoretic Framework}

The type-theoretic framework is a method for specifying LTTs using a type system such as $\mathrm{LF}^{\prime}$. It was introduced in (Luo, 2006). The LTTs specifiable in the type-theoretic framework are all defined and specified in a uniform way, but should be capable of expressing a wide range of different mathematical foundations. The type-theoretic framework is thus especially suitable as the basis for a pluralist formalisation.

One specifies an LTT within the type-theoretic framework by:

1 Declaring a number of inductive types and inductive families of types.

Besides $N$, other examples of inductive types include those of lists, vectors, trees, ordinals, dependent functions and dependent pairs. In general, inductive types can be generated by inductive schemata as studied in, for example, (Dybjer, 1991; Coquard and Paulin-Mohring, 1990; Luo, 1994).

2 Declaring a number of type universes: types that whose objects are (names of) types. For example, a universe $U$ of 'small types' can be introduced as

$$
U \text { : Type and T: (U)Type; }
$$

An inductive type may have a name in $U$ : e.g. we may have nat as a name of $N$ :

$$
\text { nat: } U \text { and } T(n a t)=N \text { : Type. }
$$

Notice that such a universe is predicative, in the sense that it only contains types that do not involve $U$ itself. The general way of introducing predicative type universes can be found in (Martin-Löf, 1984). Impredicative universes, such as that of propositions in UTT (Luo, 1994, p.175), can also be specified in the type-theoretic framework.

3 Declaring a number of logical connectives and their associated rules of deduction.

We may introduce some or all of the propositional connectives, first- or higher-order 
quantifiers, and other logical connectives (such as equality). The rules of deduction may be those of classical logic, constructive logic, minimal logic, etc.

4 Declaring one or more propositional universes (Adams and Luo, 2010).

5 Declaring the induction rules for each inductive data type.

An LTT may contain some data types and usually they are inductively defined, exactly the way as inductive types are specified in LF (see Section 3.1). For each inductive type in the LTT, there is an associated induction rule for proving properties of the objects of that type.

For example, an LTT may contain the type $N$ of natural numbers as specified in Figure 2 in Section 3.1. Associated with $N$, there is an associated induction rule given by the following constant $\mathcal{E}_{P}^{N}$ with associated rule

$$
\frac{\Gamma \vdash P:(N) \operatorname{Prop} \quad \Gamma \vdash c: P(0) \quad \Gamma \vdash f:(x: N)(P(x)) P(\operatorname{succ}(x)) \quad \Gamma \vdash n: N}{\Gamma \vdash \mathcal{E}_{P}^{N}(P, c, f, n): P(n)}
$$

Note that, when read as a rule of proof, the above is just the rule of induction over natural numbers.

Therefore, associated with each inductive type, there are two elimination operators: $\mathcal{E}_{T}$ and $\mathcal{E}_{P}$ (for $N$, they are $\mathcal{E}_{T}^{N}$ and $\mathcal{E}_{P}^{N}$ ). Note that the elimination operator over types, $\mathcal{E}_{T}$, has associated computation rules (e.g., the computation rules for $\mathcal{E}_{T}^{N}$ in Figure 2), while the elimination operator over propositions, $\mathcal{E}_{P}$, does not. ${ }^{\S}$

We may introduce rules for induction over the whole of Prop as above, or over just one propositional universe.

The induction rules connect the world of logical propositions (formally represented by Prop) and that of the data types (formally represented by Type). Quantifications over types allow one to form propositions to express logical properties of data and the induction rules to prove those properties.

6 Introducing types of typed sets.

For each type $A$, we can introduce a type $\operatorname{Set}(A)$ of all sets of objects of type $A$. This type's canonical objects have the form $\{x: A \mid \phi\}$, where $\phi$ is a proposition. This allows us to introduce sets in an impredicative way (if $\phi$ may range over the whole of Prop), as in ordinary mathematics, or a predicative way (if $\phi$ ranges only over a small universe of propositions, as in predicative mathematics; see, e.g., (Feferman, 2005)). (For further and formal details, see (Luo, 2006; Adams and Luo, 2010)).

Such a notion of typed set, together with the possibility of representing the classical first-order logic, allows us to formalise classical predicative mathematics in the typetheoretic framework (Weyl, 1918; Adams and Luo, 2010) as well as impredicative mathematics (cf., the discussion on mathematical pluralism in Section 2).

\subsubsection{Remarks}

\footnotetext{
$\S$ For the elimination operators over propositions, it is optional whether they have associated computation rules, similar to those for the elimination operators over types. Including or excluding these computation rules will not affect the type-theoretic component (since types and terms may not depend on proofs) or which propositions are provable.
} 
1 Separation of propositions and types The type-theoretic framework has an important salient feature: there is a clear separation between logical propositions and data types. In Martin-Löf's type theory, for example, types and propositions are identified. The second author has argued, for instance in the development of ECC/UTT (Luo, 1994), that it is unnatural to identify logical propositions with data types and there should be a clear distinction between the two. This is part of the philosophy behind the development of the type theories ECC and UTT, where data types are not propositions, although logical propositions are types.

Logic-enriched type theories have gone one step further (as compared with ECC/UTT) - there is a complete separation between propositions and types. Logical propositions or their totality Prop are not regarded as types. This has led to a more flexible treatment of logics.

2 Consistency and Adequacy. The consistency of an LTT formulated following the above suggestions can be shown either by a direct proof (Goguen, 1994) or by an indirect mapping between the LTT concerned to a known consistent type system. For example, in (Luo, 2006), we map an LTT called $L T T_{1}$ (classical FOL plus inductive types) to $M L T T_{e}$, an extension of Martin-Löf's type theory with the excluded middle. We show that $L T T_{1}$ is consistent relative to $M L T T_{e}$.

Such a relative consistency proof raises an interesting question: if Martin-Löf's type theory extended with excluded middle is consistent, why use an LTT at all? Why not just use $M L T T_{e}$ ? One reason is that the meaning theory of type theory relies on the property of canonicity: that every object reduces to a canonical object. This makes it possible for us to provide a meaning theory in which an inductive type is understood as consisting of its canonical objects (for example, the type of natural numbers consists of zero and its successors).

The LTTs in the type-theoretic framework possess the property of canonicity, thanks to the clear distinction between logical propositions and data types. The system $M L T T_{e}$ does not; in $M L T T_{e}$, every inductive type contains infinitely many noncanonical objects. The type-theoretic framework hence provides an adequate treatment of classical reasoning on the one hand, and a clean meaning-theoretic understanding of inductive types on the other.

\subsection{Working with More than One LTT}

We are concerned in this paper with formalisations that involve more than one LTT. We must therefore extend the logical framework yet again.

If we wish to declare two LTTs simultaneously, called $\mathrm{LTT}_{1}$ and $\mathrm{LTT}_{2}$, say, then we shall need the logical framework to possess the following kinds, besides the dependent product kinds of the form $(x: K) K^{\prime}$ :

$$
\text { Type }_{1}, \mathrm{El}_{1}(k), \operatorname{Prop}_{1}, \operatorname{Prf}_{1}(k), \quad \mathbf{T y p e}_{2}, \mathrm{El}_{2}(k), \operatorname{Prop}_{2}, \operatorname{Prf}_{2}(k)
$$

with the following rules of deduction:

$$
\frac{\Gamma \text { valid }}{\Gamma \vdash \text { Type }_{1} \text { kind }} \quad \frac{\Gamma \vdash A: \text { Type }_{1}}{\Gamma \vdash \mathrm{El}_{1}(A) \text { kind }} \quad \frac{\Gamma \text { valid }}{\Gamma \vdash \text { Prop }_{1} \text { kind }} \quad \frac{\Gamma \vdash P: \text { Prop }_{1}}{\Gamma \vdash \text { Prf }_{1}(P) \text { kind }}
$$




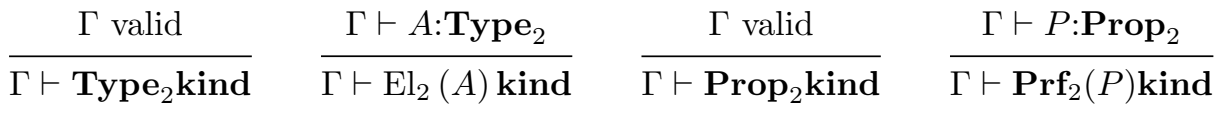

In the framework augmented with these kinds:

- the objects of kind $\mathbf{T y p e}_{i}$ represent the types of $\mathrm{LTT}_{i}$;

- the objects of kind $\mathrm{El}_{i}(A)$ represent the terms of $\mathrm{LTT}_{i}$;

- the objects of kind $\operatorname{Prop}_{i}$ represent the propositions of $\mathrm{LTT}_{i}$;

— the objects of kind $\operatorname{Prf}_{i}(A)$ represent the proofs of $\mathrm{LTT}_{i}$

The constants and computation rules in the declaration of $\mathrm{LTT}_{1}$ will therefore involve only the kinds $\mathbf{T y p e} \mathbf{P}_{1}, \mathrm{El}_{1}(A), \mathbf{P r o p}_{1}$ and $\mathbf{P r f}_{1}(A)$, and the product kinds built up from these. When we are working in $\mathrm{LTT}_{1}$, we use only these kinds. Likewise, when declaring or working in $\mathrm{LTT}_{2}$, we use only the kinds $\mathbf{T y p e}_{2}, \mathrm{El}_{2}(A), \mathbf{P r o p}_{2}, \operatorname{Prf}_{2}(A)$, and the product kinds built from these.

3.4.1. Logical Framework $\mathrm{LF}_{\mathrm{LTT}}$ We wish to give the user the ability to declare arbitrarily many pairs $(K, C)$ consisting of a top-kind $K$ and a kind constructor $C$.

The effect of declaring the pair $(K, C)$ is to extend the logical framework with the following rules of deduction:

$$
\frac{\Gamma \text { valid }}{\Gamma \vdash K \text { kind }} \quad \frac{\Gamma \vdash k: K}{\Gamma \vdash C(k) \text { kind }} \quad \frac{\Gamma \vdash k=k^{\prime}: K}{\Gamma \vdash C(k)=C\left(k^{\prime}\right)}
$$

In the above example, we would declare the pairs

$$
\left(\text { Type }_{1}, E l_{1}\right),\left(\text { Type }_{2}, E l_{2}\right),\left(\operatorname{Prop}_{1}, \operatorname{Prf}_{1}\right),\left(\operatorname{Prop}_{2}, \operatorname{Prf}_{2}\right) \text {. }
$$

We note that there is no longer a need to have the kinds Type, El $(A)$, Prop and $\operatorname{Prf}(P)$ as part of the primitive syntax. We therefore remove them. The user can reintroduce them if needed by declaring the topkind pairs (Type, El) and (Prop, Prf).

We call this new framework $L_{\mathrm{LTT}}$. In summary: $\mathrm{LF}_{\mathrm{LTT}}$ is the framework LF, with the kinds Type and $E l(k)$ removed, and with the ability to declare pairs $(K, C)$ added.

\subsection{Implementation}

The proof assistant Plastic was first implement by Callaghan as an implementation of LF (Callaghan and Luo, 2001). It was extended to an implementation of $L F^{\prime}$, and used in the work to formalise Weyl's predicative mathematics using the type-theoretic framework (Adams and Luo, 2007; Adams and Luo, 2010).

Plastic allows the user to declare constants with commands such as $c: K$ by $[c: K]$; to define constants with commands such as $[c=\cdots: K]$; and to construct objects using tactics such as Intros and Refine. The user may also declare computation rules of a certain form. Plastic automatically generates the constants and computation rules for inductive types, but logical connectives, induction rules, universes and computation rules in the logical component must be entered by hand.

For the work described in this paper, the first author is extending Plastic further to an 
implementation of $\mathrm{LF}_{\mathrm{LTT}}$. The user may now declare a top-kind and constructor $(K, C)$ by entering the command Topkind $K C$;

We shall describe the Plastic implementations of several LTTs in Section 5 and how they are used in studying pluralist formalisations and proof reuse.

\section{Our Approach to Pluralist Formalisations}

Our approach to pluralist formalisations is based on the concept of a translation:

Definition 4.1 (Translation). A translation $\Phi$ from an LTT $S$ to an LTT $T$ is a mapping from the expressions of $S$ to the expressions of $T$ such that:

- if $A$ is a type of $S$, then $\Phi(A)$ is a type of $T$;

- if $M$ is a term of type $A$ in $S$, then $\Phi(M)$ is a term of type $\Phi(A)$ in $T$;

- if $P$ is a proposition of $S$, then $\Phi(P)$ is a proposition of $T$;

- if $H$ is a proof of $P$ in $S$, then $\Phi(H)$ is a proof of $\Phi(P)$ in $T$.

Suppose we have a translation $\Phi$ from $S$ to $T$. If $\alpha$ is a theorem of $S$, then $\Phi(\alpha)$ is a theorem of $T$. We wish to find a way to take a formalisation of a proof of $\alpha$ in $S$, and use that proof script - without any modification - as a proof of $\Phi(\alpha)$ in $T$.

Our approach is as follows(see Fig. 1). Let $M_{S}$ and $M_{T}$ be two proof scripts that declare the constants and rules of deduction of $S$ and $T$ respectively. Let $M_{\alpha}$ be a proof script that imports $M_{S}$, and proves the theorem $\alpha$.

We construct a proof script $M_{\phi}$ that imports $M_{T}$, and then defines every symbol that was declared as a constant in $M_{S}$. If $M_{S}$ contains a constant declaration $c: K$, then $M_{T}$ must define $c$ to be an object of kind $K$. If $M_{S}$ declares the computation rule $M \triangleright N$, then we must ensure that $M$ and $N$ are convertible under the definitions in $M_{T}$.

If both these conditions are met, then we know that the proof script $M_{\alpha}$ will parse if we import $M_{\phi}$ instead of $M_{S}$. Let us give the name $M_{\phi(\alpha)}$ to the proof script with this small change made (see Fig. 1).

Example 4.2. Let $\mathrm{LTT}_{1}$ be an LTT whose logical component consists of classical propositional logic with negation not and implication imp. Let $\mathrm{LTT}_{2}$ be an LTT whole logical component consists of classical propositional logic with negation neg and disjunction or (see Fig. 4). We shall write $\sim \phi$ for not $\phi, \phi \supset \psi$ for $\operatorname{imp} \phi \psi, \neg \phi$ for neg $\phi$, and $\phi \vee \psi$ for or $\phi \psi$.

It is known that implication can be defined in terms of disjunction and negation in classical logic. This fact can be used to define a translation from $\mathrm{LTT}_{1}$ to $\mathrm{LTT}_{2}$ :

$$
\llbracket \sim \phi \rrbracket \equiv \neg \llbracket \phi \rrbracket \quad \llbracket \phi \supset \psi \rrbracket \equiv \neg \llbracket \phi \rrbracket \vee \llbracket \psi \rrbracket
$$

Let $M_{1}$ and $M_{2}$ be two proof scripts that consist of the constant declarations given in Fig. 4 for $\mathrm{LTT}_{1}$ and $\mathrm{LTT}_{2}$ respectively. We now wish to write a proof script $M_{\Phi}$ that imports $M_{2}$, and then defines every constant that was declared in $M_{1}$.

The proof script $M_{\phi}$ begins as follows: 


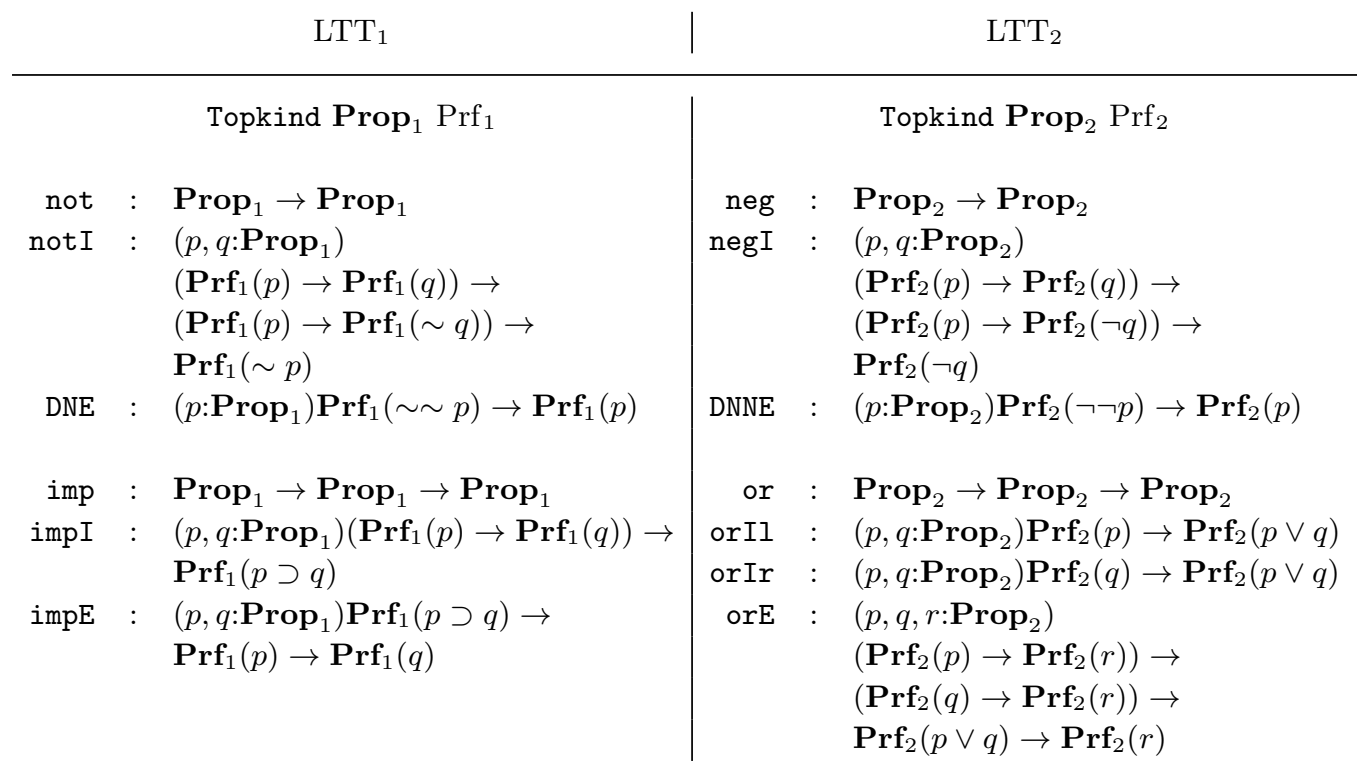

Fig. 4. The logical components of $\mathrm{LTT}_{1}$ and $\mathrm{LTT}_{2}$

$$
\begin{aligned}
& \text { import } M_{2} ; \\
& {\left[\text { Prop }_{1}=\text { Prop }_{2}\right] ;} \\
& {\left[\operatorname{Prf}_{1}=\operatorname{Prf}_{2}\right] ;} \\
& {[\text { not }=\text { neg]; }} \\
& {[\text { not I }=\text { negI }] ;} \\
& {[\text { DNE }=\text { DNNE }] ;}
\end{aligned}
$$

We must now define imp. The definition is guided by (1) above:

$$
\left[\mathrm{imp}=\left[\mathrm{p}, \mathrm{q}: \mathbf{P r o p}_{1}\right] \sim \mathrm{p} \vee \mathrm{q}\right] ;
$$

We must now define objects impI and impE that have kinds

$$
\begin{aligned}
& \text { impI } \quad: \quad\left(p, q: \operatorname{Prop}_{1}\right)\left(\operatorname{Prf}_{1}(p) \rightarrow \operatorname{Prf}_{1}(q)\right) \rightarrow \operatorname{Prf}_{1}(p \supset q) \\
& \text { impE : }\left(p, q: \mathbf{P r o p}_{1}\right) \operatorname{Prf}_{1}(p \supset q) \rightarrow \operatorname{Prf}_{1}(p) \rightarrow \operatorname{Prf}_{1}(q) \\
& \text { i.e. } \quad \text { impI : }\left(p, q: \operatorname{Prop}_{2}\right)\left(\operatorname{Prf}_{2}(p) \rightarrow \operatorname{Prf}_{2}(q)\right) \rightarrow \operatorname{Prf}_{2}(\sim p \vee q) \\
& \text { impE }:\left(p, q: \mathbf{P r o p}_{2}\right) \mathbf{P r f}_{2}(\sim p \vee q) \rightarrow \operatorname{Prf}_{2}(p) \rightarrow \operatorname{Prf}_{2}(q)
\end{aligned}
$$

It is straightforward to construct these objects using a proof assistant like Plastic.

Now, suppose we have formalised a proof of the proposition $\alpha$ in $\mathrm{LTT}_{2}$. That is, suppose we have a proof script that imports $\mathbf{M}_{2}$, and then constructs an object of kind $\mathbf{P r f}_{2}(\alpha)$. If we change the script so it imports $M_{\Phi}$ instead, then we know that the script shall still parse; and the script shall now be a proof of $\llbracket \alpha \rrbracket$ in $\mathrm{LTT}_{2}$.

For example, suppose that a script imports $M_{2}$ that constructs an object of kind 
$\left(p: \operatorname{Prop}_{2}\right) \operatorname{Prf}_{2}(p \supset p)$. We change the script to import $M_{\Phi}$ instead. Under the definitions in $M_{\Phi}$, we have

$$
\left(p: \operatorname{Prop}_{2}\right) \operatorname{Prf}_{2}(p \supset p)=\left(p: \operatorname{Prop}_{1}\right) \operatorname{Prf}_{1}(\sim p \vee p)
$$

and so the script now constructs an object of kind $\left(p: \mathbf{P r o p}_{1}\right) \operatorname{Prf}_{1}(\sim p \vee p)$. This is exactly as required, since $\llbracket \phi \supset \phi \rrbracket \equiv \sim \llbracket \phi \rrbracket \vee \llbracket \phi \rrbracket$.

\subsection{Remarks}

1 Note that the construction of the module $M_{\Phi}$ can be seen in one sense as a formalisation of the metatheorem that $\llbracket \rrbracket$ is sound, i.e. maps theorems to theorems.

2 Note that a translation between two LTTs may involve changing the logical world (as in the examples in Sections 5.2-5.4 below), the datatype world (e.g. the inclusion from $L T T_{W}$ to $L T T_{I}$ considered in (Adams and Luo, 2010)) or both.

\section{Case Studies in Formalisation}

We now describe several case studies in the use of this method of proof reuse that we have carried out using the proof assistant Plastic. The source code for these examples is available at http://www.cs.rhul.ac.uk/ robin/pluralism

\subsection{Classical and Intuitionistic LTTs}

For these examples, we shall assume that we have two LTTs: a classical LTT, LTT class $_{\text {, }}$ and an intuitionistic LTT, LTT int. We assume that these two LTTs have the same typetheoretic component. The logical component of $\mathrm{LTT}_{\text {class }}$ is first-order classical logic with the connectives eqC, notC, andC, orC, impC, allC and exC. The logical component of $\mathrm{LTT}_{\text {int }}$ is first-order intuitionistic logic with the connectives eqI, notI, andI, orI, impI, alli and exI.

We write $M={ }_{C} N$ for eqC $A M N, \neg_{C} \phi$ for notC $\phi, \phi \wedge_{C} \psi$ for andC $\phi \psi, \phi \vee_{C} \psi$ for $\operatorname{orC} \phi \psi, \phi \supset_{C} \psi$ for impC $\phi \psi, \forall_{C} x: A$. $\phi$ for allC $A[x: A] \phi$, and $\exists_{C} x: A$. $\phi$ for exC $A[x: A] \phi$.

Similarly, we write $M={ }_{I} N$ for eqI $A M N$, etc.

We have two proof scripts: $M_{C}$ that declares the constants and computation rules of $\mathrm{LTT}_{\text {class }}$, and $M_{I}$ that declares the constants and computation rules of $\mathrm{LTT}_{\mathrm{int}}$. Some of the declarations are given in Fig. 5. Note that, apart from the different names for the constants, the only difference between the two scripts is the inclusion of $\neg_{C} \neg_{C} E$ in $M_{C}$.

We shall omit the subscripts ${ }_{C}$ and ${ }_{I}$ when no confusion may result.

\subsection{Inclusion}

If an LTT $L_{1}$ is a subsystem of the LTT $L_{2}$, then we can easily reuse proof scripts from $L_{1}$ as proof scripts in $L_{2}$. The mapping $\Phi$ here is the inclusion mapping.

For example, $\mathrm{LTT}_{\mathrm{int}}$ is a subsystem of $\mathrm{LTT}_{\text {class }}$. We can easily write a module $M_{\text {ItoC }}$ 


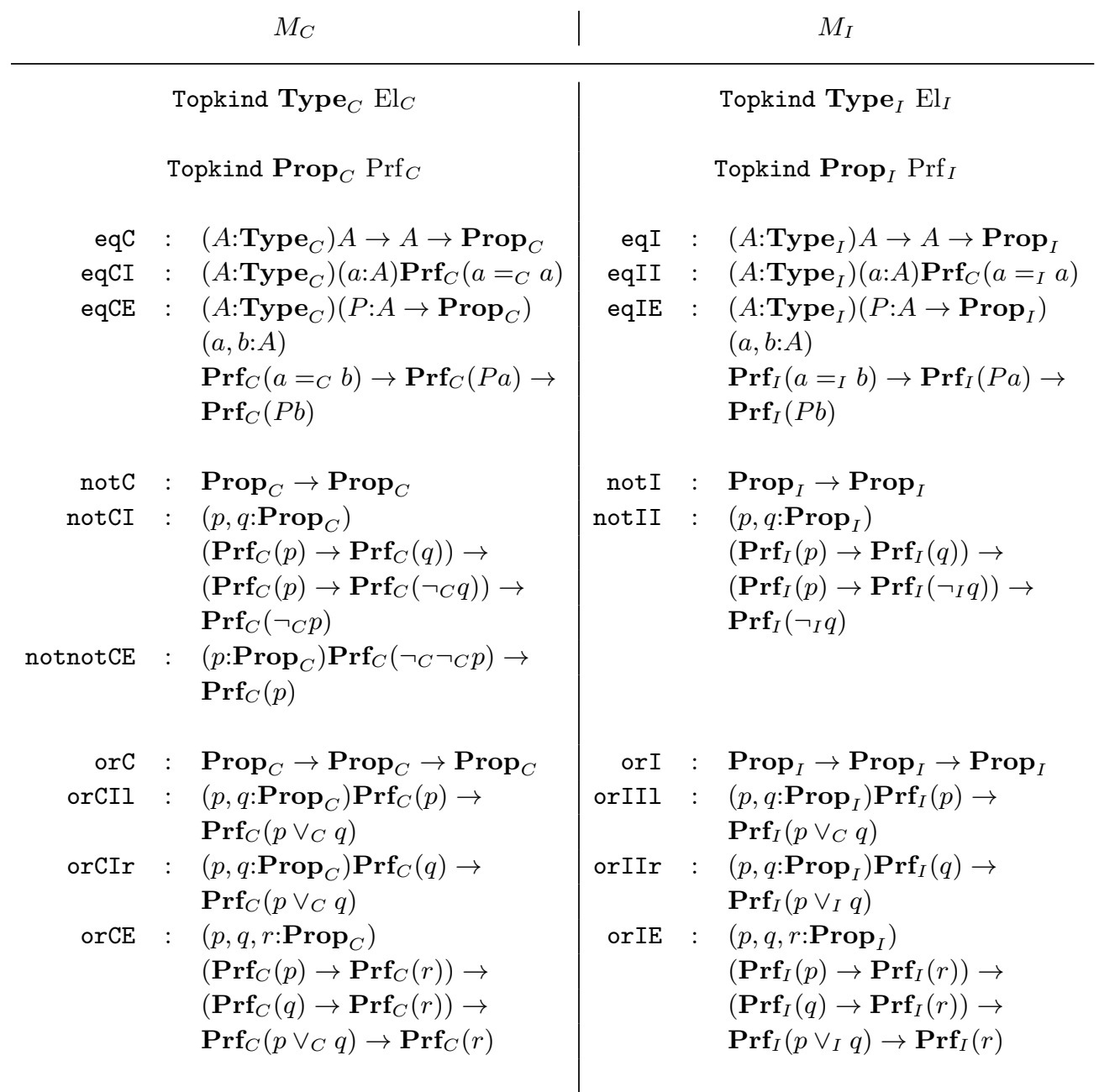

Fig. 5. The partial scripts that declare $\mathrm{LTT}_{\text {class }}$ and $\mathrm{LTT}_{\text {int }}$

that describes the inclusion mapping (Fig. 6). Any code that parses under $M_{C}$ will also parse under $M_{\text {ItoC }}$.

This form of proof reuse was used in the formalisation of Weyl's predicative foundation of mathematics (Adams and Luo, 2010). That formalisation involved two LTTs: the predicative $\mathrm{LTT}_{\mathrm{W}}$ and an impredicative extension. We defined the real numbers in $\mathrm{LTT}_{\mathrm{W}}$, proved in LTT $_{\mathrm{W}}$ the theorem that every set of rationals bounded above has a (real) least upper bound, and then reused that proof to prove in the impredicative LTT that every set of reals bounded above has a least upper bound. 


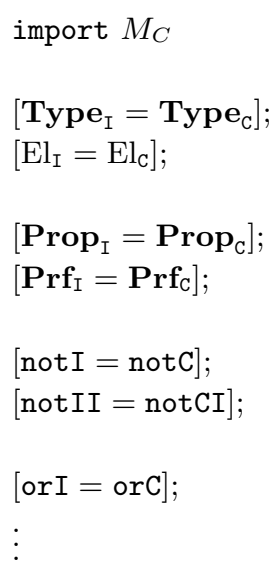

Fig. 6. The Module $M_{I t o C}$

\subsection{The Double Negation Translation}

The double negation translation, or Gödel-Gentzen negative translation (Gödel, 1933) is a mapping from classical logic to intuitionistic logic.

The mapping is defined thus:

$$
\begin{aligned}
\alpha\urcorner\urcorner & \equiv \neg \neg \alpha \quad \text { ( } \alpha \text { atomic) } \\
(\neg \phi)\urcorner\urcorner & \equiv \neg \phi\urcorner\urcorner \\
(\phi \wedge \psi)\urcorner\urcorner & \equiv \phi\urcorner\urcorner \wedge \psi\urcorner\urcorner \\
(\phi \vee \psi)\urcorner\urcorner & \equiv \neg(\neg \phi\urcorner \neg \wedge \neg \psi\urcorner\urcorner) \\
(\phi \rightarrow \psi)\urcorner\urcorner & \equiv \phi\urcorner \neg \rightarrow \psi\urcorner\urcorner \\
(\forall x \phi)\urcorner\urcorner & \equiv \forall x \phi\urcorner\urcorner \\
(\exists x \phi)\urcorner\urcorner & \equiv \neg \forall x \neg \phi\urcorner\urcorner
\end{aligned}
$$

To prove the soundness of the double negation translation, the most important step is the following lemma, which is proved by induction on $\phi$ :

Lemma 5.1. For any formula $\phi$, the formula $\phi\urcorner\urcorner$ is stable; that is, $\neg \neg \phi\urcorner \neg \supset \phi\urcorner \neg$ is provable in intuitionistic logic.

It is then quite straightforward to prove that, if $\phi$ is provable in classical logic, then $\phi\urcorner\urcorner$ is provable in intuitionistic logic, by induction on the derivation of $\phi$. We wish to write a proof script $M_{D N}$ that imports $M_{I}$ and then defines every constant that was declared in $M_{C}$.

5.3.1. First Attempt For our first attempt, we simply define

$$
\begin{aligned}
& {\left[\mathbf{T y p e}_{\mathrm{C}}=\mathbf{T y p e}_{\mathrm{I}}\right] ;} \\
& {\left[\mathrm{El}_{\mathrm{C}}=\mathrm{El}_{\mathrm{I}}\right] ;} \\
& {\left[\mathbf{P r o p}_{\mathrm{C}}=\mathbf{P r o p}_{\mathrm{I}}\right] ;} \\
& {\left[\mathbf{P r f}_{\mathrm{C}}=\mathbf{P r f}_{\mathrm{I}}\right] ;}
\end{aligned}
$$


and then define eqC, notC, etc. as follows:

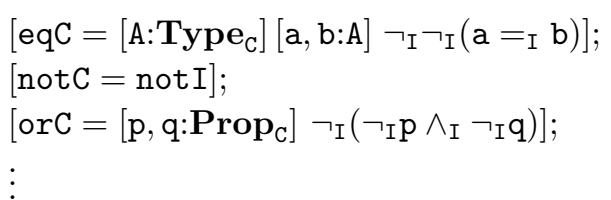

This module 'maps' formulas of $\mathrm{LTT}_{\text {class }}$ to their double-negation translation, in the following sense: an expression that denotes an proposition $\phi$ in $\mathrm{LTT}_{\text {class }}$ will expand, under these definitions, to an expression that denotes $\phi\urcorner\urcorner$ in $\mathrm{LTT}_{\text {int }}$. For example, the expression $P \vee_{C} \neg_{C} P$ expands under the above definitions to $\left.\neg_{I}\left(\neg_{I} P \wedge_{I} \neg_{I}\right\urcorner_{I} P\right)$.

However, this script will not work, as the kind of orCE is then (omitting the $\operatorname{Prf}_{I} \mathrm{~s}$ ):

$$
\left(P, Q, R: \text { Prop }_{I}\right)(P \rightarrow R) \rightarrow(Q \rightarrow R) \rightarrow \neg(\neg P \wedge \neg Q) \rightarrow R
$$

and this kind is uninhabited in $\mathrm{LTT}_{\text {int }}$.

This is because the corresponding rule of deduction

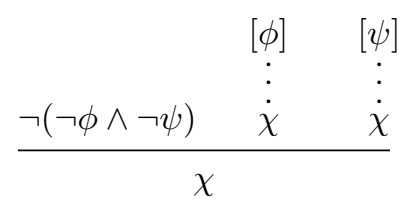

is not admissible in intuitionistic logic.

Looking at the proof of soundness, we see that we somehow need to use the fact that $\phi\urcorner\urcorner$ is always stable (Lemma 5.1). This gives us the idea for our second and successful attempt:

5.3.2. Second Attempt We must map Prop $_{C}$, not to the kind of all propositions, but to the kind of all stable propositions. Ideally, we would like to write

$$
\left.\operatorname{Prop}_{C}=\Sigma p: \operatorname{Prop}_{I} . \operatorname{Prf}_{I}\left(\neg_{I}\right\urcorner_{I} p \supset_{I} p\right) .
$$

However, $\mathrm{LF}_{\mathrm{LTT}}$ does not at present have these $\Sigma$-kinds.

One option would be to extend $\mathrm{LF}_{\text {LTT }}$ with $\Sigma$-kinds, or some similar feature. This is an option that the authors intend to explore in the future.

As an alternative, we instead declare in $M_{D N}$ the kind $\operatorname{Prop}_{C}$, the constructor $\operatorname{Prf}_{C}$, and the following introduction, elimination and computation rules:

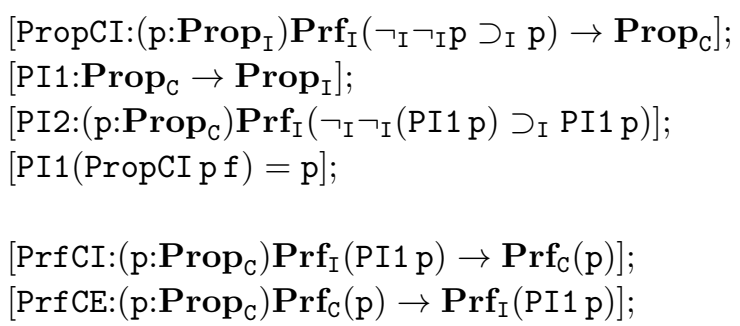

The constructor $\operatorname{Prf}_{C}$ is defined as follows:

$\left[\operatorname{Prf}_{\mathrm{C}}=\left[\mathrm{p}: \operatorname{Prop}_{\mathrm{C}}\right] \operatorname{Prf}_{\mathrm{I}}(\mathrm{PI} 1 \mathrm{p})\right]$; 
We can now proceed to define the constants of $M_{C}$. The connective $\vee_{C}$, for example, must now be defined as a binary function on this 'Sigma-kind':

$$
\begin{aligned}
\text { orC } & : \quad \operatorname{Prop}_{C} \rightarrow \operatorname{Prop}_{C} \rightarrow \text { Prop }_{C} \\
(p, f) \vee_{C}(q, g) & \equiv\left(\neg_{I}\left(\neg_{I} p \wedge_{I} \neg_{I} q\right), h\right)
\end{aligned}
$$

where $h$ is a proof that $\neg_{I}\left(\neg_{I} p \wedge_{I} \neg_{I} q\right)$ is stable.

Written out in full, we have

$$
\operatorname{orC}=\left[p, q: \mathbf{P r o p}_{C}\right] \operatorname{Prop}_{C} I\left(\neg_{I}\left(\neg_{I}(P I 1 p) \wedge_{I} \neg_{I}(P I 1 q)\right) \cdots\right.
$$

where $\cdots$ elides a proof of

$$
\neg \neg \neg(\neg(P I 1 p) \wedge \neg(P I 1 q)) \supset \neg(\neg(P I 1 p) \wedge \neg(P I 1 q)) .
$$

The kind of orCE expands under the above definitions to

$$
\begin{gathered}
\left(P, Q, R: \operatorname{Prop}_{C}\right)\left(\operatorname{Prf}_{I}\left(P_{1}\right) \rightarrow \operatorname{Prf}_{I}\left(R_{1}\right)\right) \rightarrow\left(\operatorname{Prf}_{I}\left(Q_{1}\right) \rightarrow \operatorname{Prf}_{I}\left(R_{1}\right)\right) \rightarrow \\
\operatorname{Prf}_{I}\left(\neg_{I}\left(\neg_{I} P_{1} \wedge_{I} \neg_{I} Q_{1}\right)\right) \rightarrow \operatorname{Prf}_{I}\left(R_{1}\right)
\end{gathered}
$$

which is inhabited. The inhabitant we construct makes use of $R_{2}: \operatorname{Prf}_{I}\left(\neg_{I} \neg_{I} R_{1} \supset_{I} R_{1}\right)$.

It is possible to define every constant in $M_{C}$ in this fashion. Any module that imports $M_{C}$ will parse if it is changed to import $M_{D N}$ instead. We have:

- If an expression denotes a proposition $\phi$ under $M_{C}$, then under $M_{D N}$ it denotes a pair consisting of $\phi\urcorner\urcorner$ and a proof that $\phi\urcorner\urcorner$ is stable.

- If an expression denotes a proof $P$ of $\phi$ under $M_{C}$ (i.e. $\left.P: \operatorname{Prf}_{C}(\phi)\right)$, then under $M_{D N}$ it denotes a proof of $\phi\urcorner\urcorner$.

5.3.3. Application As an application of this work, we can show that, if $\mathrm{LTT}_{\text {int }}$ is consistent, then $\mathrm{LTT}_{\text {class }}$ is consistent. Suppose we had a proof script that imports $M_{C}$, and then constructs an object of type $\operatorname{Prf}_{C}\left(\perp_{C}\right)$. Then the same proof script could import $M_{D N}$ instead, in which case it would construct an object of type $\operatorname{Prf}_{I}\left(\perp_{I}\right)$.

\subsection{The A-translation}

The $A$-translation (Friedman, 1978) is a mapping from intuitionistic logic to intuitionistic logic. We fix a formula $A$, and then define the formula $\phi^{A}$ for every formula $\phi$ as follows:

$$
\begin{array}{rlrl}
P^{A} & \equiv P \vee A & (P \text { atomic }) \\
(\neg \phi)^{A} & \equiv \phi^{A} \supset A & \\
(\phi * \psi)^{A} & \equiv \phi^{A} * \psi^{A} & (* \equiv \wedge, \vee, \supset) \\
(Q x \phi)^{A} & \equiv Q x \phi^{A} \quad(Q \equiv \forall, \exists)
\end{array}
$$

This translation is sound: if $\phi$ is a theorem of an intuitionistic theory $T$, then so is $\phi^{A}$ (Friedman, 1978). The important lemma in the proof of this theorem is:

Lemma 5.2. For any formula $\phi$, we have $\vdash A \rightarrow \phi^{A}$. 
We can make use of the $A$-translation for proof reuse as follows. We write two copies of a script that defines $\operatorname{LTT}_{\text {int }}$, say $M_{I}$ and $M_{I}^{\prime}$ :

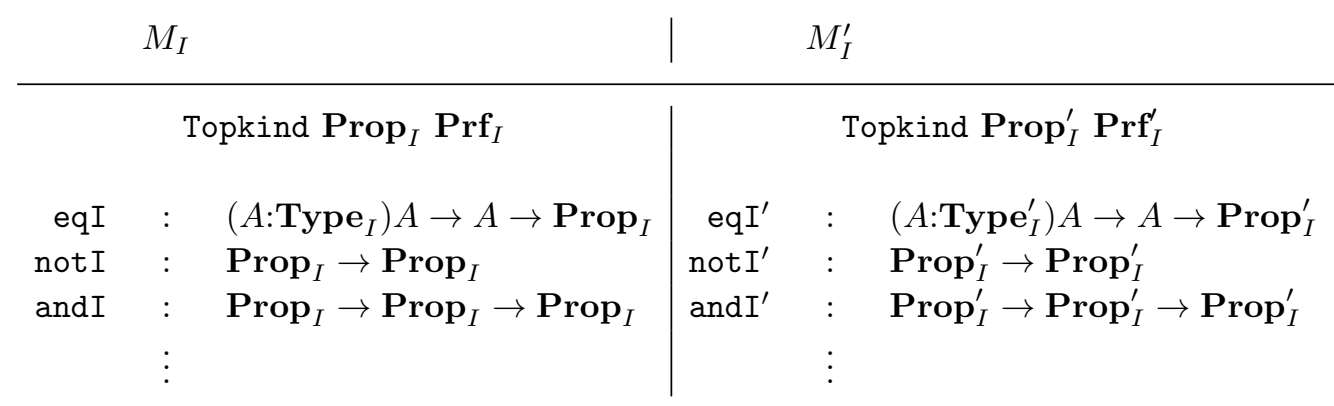

We construct our module $M_{A}$ that defines the $A$-translation as follows. We assume that $M_{I}$ has been imported and an object $A: \mathbf{P r o p}_{I}$ defined. We now define every constant declared in $M_{I}^{\prime}$. The constant $\mathbf{P r o p}_{I}^{\prime}$ is defined to be the kind of all propositions $\phi: \mathbf{P r o p}_{I}$ such that $A \supset \phi$. Again, we would like to write:

$$
\begin{aligned}
\operatorname{Prop}_{I}^{\prime} & =\Sigma p: \mathbf{P r o p}_{I} \cdot \mathbf{P r f}_{I}\left(A \supset_{I} p\right) \\
\operatorname{Prf}_{I}^{\prime}(\phi, f) & =\operatorname{Prf}_{I}(\phi) \\
a={ }_{I}^{\prime} b & =\left(a=_{I} b \vee_{I} A, \cdots\right) \\
\neg_{I}^{\prime}(\phi, f) & =\left(\phi \supset_{I} A, \cdots\right) \\
(\phi, f) \vee_{I}^{\prime}(\psi, g) & =\left(\phi \vee_{I} \psi, \cdots\right)
\end{aligned}
$$

But as $L F_{L T T}$ does not have $\Sigma$-kinds, we instead declare the kind Prop $\mathbf{P}_{I}^{\prime}$, the constructor $\operatorname{Prf}_{I}^{\prime}$, and the following constants:

$$
\begin{aligned}
& \operatorname{Prop}_{I}^{\prime} I \quad: \quad\left(p: \operatorname{Prop}_{I}\right) \operatorname{Prf}_{I}\left(A \supset_{I} p\right) \rightarrow \operatorname{Prop}_{I}^{\prime} \\
& P I 1 \quad: \quad \operatorname{Prop}_{I}^{\prime} I \rightarrow \operatorname{Prop}_{I} \\
& P I 2 \quad: \quad\left(p: \operatorname{Prop}_{I}^{\prime}\right) \operatorname{Prf}_{I}\left(A \supset_{I} P I 1 p\right) \\
& \operatorname{Prf}_{I} 2 \operatorname{Prf}_{I}^{\prime} \quad: \quad\left(p: \operatorname{Prop}_{I}\right)\left(f: \operatorname{Prf}_{I}\left(A \supset_{I} p\right)\right) \operatorname{Prf}_{I}^{\prime}\left(\mathbf{P r o p}_{I}^{\prime} I p f\right) \rightarrow \operatorname{Prf}_{I}(p) \\
& \operatorname{Prf}_{I}^{\prime} 2 \operatorname{Prf}_{I} \quad: \quad\left(p: \operatorname{Prop}_{I}\right)\left(f: \operatorname{Prf}_{I}\left(A \supset_{I} p\right)\right) \operatorname{Prf}_{I}(p) \rightarrow \operatorname{Prf}_{I}^{\prime}\left(\operatorname{Prop}_{I}^{\prime} I p f\right) \\
& a={ }_{I}^{\prime} b=\operatorname{Prop}_{I}^{\prime} I\left(a={ }_{I} b \vee_{I} A\right)(\cdots) \\
& \neg_{I}^{\prime} p=\operatorname{Prop}_{I}^{\prime} I\left(P I 1 p \supset_{I} A\right)(\cdots) \\
& p \vee_{I}^{\prime} q=\operatorname{Prop}_{I}^{\prime} I\left(P I 1 p \vee_{I} P I 1 q\right)(\cdots)
\end{aligned}
$$

and the computation rule $P I 1\left(\mathbf{P r o p}_{I}^{\prime} I p f\right)=p$ :Prop ${ }_{I}$.

Now any proof script beginning import $M_{I}^{\prime}$; will also parse if we replace this line with

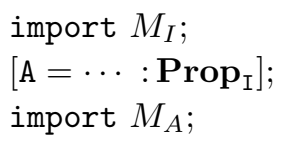

As an application of the $A$-translation, we can show that Markov's law is admissible for quantifier-free formulas: 
Theorem 5.3. Let $T$ be an intuitionistic theory. If $T \vdash \neg \neg \exists x \phi$, where $\phi$ is quantifier-free (possibly with free variables other than $x$ ), then $T \vdash \exists x \phi$.

Proof. Let $A \equiv \exists x \phi$. If $T \vdash \neg \neg \exists x \phi$ then, by the soundness of the $A$-translation, $T \vdash\left(\exists x \phi^{A} \rightarrow A\right) \rightarrow A$.

Now, it is easy to show that $\phi^{A} \vdash \phi \vee A$ and $\phi \vee A \vdash \phi^{A}$ for $\phi$ quantifier-free. Thus, we have $T \vdash(\exists x(\phi \vee A) \rightarrow A) \rightarrow A$. We have $\exists x \phi \rightarrow A$ and $\exists x A \rightarrow A$, hence $T \vdash \exists x(\phi \vee A) \rightarrow A$, and so $T \vdash A$; i.e. $T \vdash \exists x \phi$.

We can make use of this result as follows: given any proof of $\neg_{I}^{\prime} \neg_{I}^{\prime} \exists x \phi$ under $M_{I}^{\prime}$, we can obtain a proof of $\exists x \phi$ under $M_{I}$.

Suppose we have a proof script $M_{\alpha}$ that imports $M_{I}^{\prime}$ and proves $\neg \neg \exists x \phi$ :

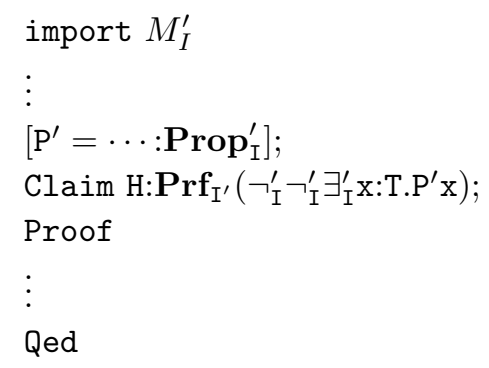

Then we can produce a proof script that imports $M_{I}$ and proves $\exists x \phi$ :

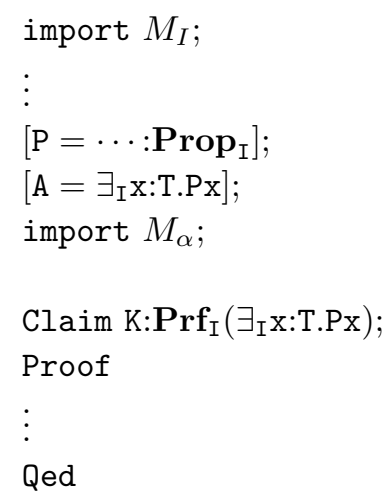

In this script, the line defining $P$ (line 3 ) is the result of replacing $\wedge_{I^{\prime}}$ with $\wedge_{I}, \vee_{I^{\prime}}$ with $\vee_{I}$, etc. in line 6 .

The proof $K$ makes use of $H$, which is now a proof of $\left(\left(\exists_{I} x: T .\left(P x \vee_{I} A\right)\right) \supset_{I} A\right) \supset_{I} A$.

5.4.1. Remark It is unsatisfactory to have to work in two copies of $\mathrm{LTT}_{\text {int }}$, and to have separate definitions of $P$ and $P^{\prime}$. We would like to be able to take a script that proves $\neg \neg \exists x P x$ in $L_{T T}$ int, and produce a script that proves $\exists x P x$ in $\mathrm{LTT}_{\text {int }}$. This requires a more sophisticated module mechanism than the one that currently exists in Plastic. 


\subsection{The Russell-Prawitz Modality}

The following mapping from a first-order language to a second-order language, first introduced in (Russell, 1903), was given the name of the Russell-Prawitz modality by Aczel (Aczel, 2001).

$$
\begin{aligned}
\llbracket P \rrbracket & \equiv P \quad(P \text { atomic }) \\
\llbracket \neg \phi \rrbracket & \equiv \forall p \cdot \llbracket \phi \rrbracket \supset p \\
\llbracket \phi \wedge \psi \rrbracket & \equiv \forall p \cdot \llbracket \phi \rrbracket \supset \llbracket \psi \rrbracket \supset p \\
\llbracket \phi \vee \psi \rrbracket & \equiv \forall p \cdot(\llbracket \phi \rrbracket \supset p) \supset(\llbracket \psi \rrbracket \supset p) \supset p \\
\llbracket \phi \supset \psi \rrbracket & \equiv \llbracket \phi \rrbracket \supset \llbracket \psi \rrbracket \\
\llbracket \forall x \phi \rrbracket & \equiv \forall x \llbracket \phi \rrbracket \\
\llbracket \exists x \phi \rrbracket & \equiv \forall p \cdot(\forall x \llbracket \phi \rrbracket \supset p) \supset p
\end{aligned}
$$

This mapping can easily be turned into a mapping between two LTTs, which can be handled by our method.

Let $\mathrm{LTT}_{1}$ be a first-order LTT with connectives not1, and1, or1, imp1, all1, ex1. Let $\mathrm{LTT}_{2}$ be a second-order LTT with connective imp2, a first-order quantifier al12: $\left(A: \mathbf{T y p e}_{2}\right)(A \rightarrow$ Prop $\left._{2}\right) \rightarrow$ Prop $_{2}$, and a second-order quantifier All2: $\left(\right.$ Prop $_{2} \rightarrow$ Prop $\left._{2}\right) \rightarrow$ Prop $_{2}$.

We write $\phi \supset_{2} \psi$ for imp2 $\phi \psi, \forall_{2} x: A$. $\phi$ for all2 $A[x: A] \phi$, and $\forall_{2} p$ :Prop Pror $_{2}$. for $\mathrm{All2}\left[p: \mathbf{P r o p}_{2}\right] \phi$.

Let $M_{1}$ and $M_{2}$ be two proof scripts that declare these two LTTs.

We can write a module $M_{R P}$ which imports $M_{2}$, and then defines every constant declared in $M_{1}$ :

$$
\begin{aligned}
& \text { Type }_{1}=\text { Type }_{2} \\
& \text { Prop }_{1}=\text { Prop }_{2} \\
& \operatorname{Prf}_{1}=\operatorname{Prf}_{2} \\
& \text { not1 }=\left[p: \text { Prop }_{1}\right] \forall q: \operatorname{Prop}_{2} \cdot p \supset_{2} q \\
& \text { and1 }=\left[p, q: \text { Prop }_{1}\right] \forall r: \text { Prop }_{2} \cdot p \supset_{2} q \supset_{2} r \\
& \text { or1 }=\left[p, q: \mathbf{P r o p}_{1}\right] \forall r: \mathbf{P r o p}_{2} \cdot\left(p \supset_{2} r\right) \supset_{2}\left(q \supset_{2} r\right) \supset_{2} r \\
& \text { imp1 = imp2 } \\
& \text { all1 }=\text { all2 } \\
& \text { ex1 }=\left[A: \text { Type }_{1}\right]\left[P: A \rightarrow \text { Prop }_{1}\right] \forall_{2} p: \text { Prop }_{2} \cdot\left(\forall_{2} x: A . P x \supset_{2} p\right) \supset_{2} p
\end{aligned}
$$

Thus, our method of proof reuse can be applied to the Russell-Prawitz modality.

\subsection{Other Applications}

Our formalisation examples discussed in this paper have all concentrated on translations that redefine the logical connectives, while leaving the world of data types unchanged. As we mentioned above (cf., the remark at the end of Section 4), the LTT-approach to 
reuse also allows the possibility of changing the world of data types in formalisations. An example of such is to reuse the results in the formalisation of predicative mathematics in the formalisation of impredicative mathematics; this is studied in (Adams and Luo, 2010).

There could be further applications. Here are some possibilities.

1 Translations that redefine the type constructors.

For example, there is a translation from System $\mathrm{T}$ to System $\mathrm{F}$ described in (Girard et al., 1990):

$$
\begin{aligned}
\llbracket B o o l \rrbracket & =\Pi X . X \rightarrow X \rightarrow X \\
\llbracket N a t \rrbracket & =\Pi X . X \rightarrow(X \rightarrow X) \rightarrow X \\
\llbracket A \rightarrow B \rrbracket & =\llbracket A \rrbracket \rightarrow \llbracket B \rrbracket \\
\llbracket A \times B \rrbracket & =\Pi X .(\llbracket A \rrbracket \rightarrow \llbracket B \rrbracket \rightarrow X) \rightarrow X
\end{aligned}
$$

Our method of proof reuse can be applied in this case. The module declaring System T will declare constants Bool:Type, Nat:Type, $\rightarrow$ :Type $\rightarrow$ Type $\rightarrow$ Type and $\times:$ Type $\rightarrow$ Type $\rightarrow$ Type. The module $M_{\Phi}$ will redefine these constants.

2 If we have two first-order systems $S$ and $T$, and every axiom of $S$ is a theorem of $T$, then every theorem of $S$ is a theorem of $T$.

Our method of proof reuse can be applied in this case. For each axiom $\alpha$ of $S$, the module $M_{S}$ will contain a constant declaration $c_{\alpha}: \operatorname{Prf}(\alpha)$. The corresponding line in $M_{\Phi}$ will be a proof of $\alpha$ in $T$.

Our method does not appear able to handle interpretations between first-order theories, however. An interpretation (in the sense of (Shoenfield, 1967)) between $S$ and $T$ maps (e.g.) a unary function symbol $f$ of $S$ to a formula $\phi[x, y]$ of $T$ such that $T \vdash \forall x \exists ! y \phi[x, y]$.

This does not fit into the pattern of the translations above, and there is no obvious way to adapt our method to this situation without changing the theories themselves (e.g. by adding a unique choice operator).

\section{Conclusion}

We have demonstrated an original method for proof reuse when conducting formalisations that make use of more than one LTT. For some translations $\Phi$ between an LTT $S$ and an LTT $T$, it is possible to write a module $M_{\Phi}$ such that, if a proof script imports $M_{S}$ and proves $\alpha$, then changing the script to import $M_{\Phi}$ will give a proof of $\Phi(\alpha)$ in $T$.

Our case studies reported in this paper are all about the logical components. As we have pointed out in various places in the paper that it is possible to consider translations that change the part of data types (e.g., changing from predicative mathematics to impredicative mathematics). We expect further development in this respect: further cases should be studied to investigate how such non-logical changes can be used in practice.

This method should be quite general, and applicable to work in type theories and other systems of logic. However, if one wishes to formalise a large piece of mathematics that involves proving results in several different foundations, then LTTs would seem to be 
particularly suitable, as they allow for a uniform presentation and treatment of a wide range of different foundations.

For our future work, we wish to formalise one such piece of mathematics, using Plastic and this method of proof reuse.

\subsection{Related Work}

There has been considerable work on proof reuse in recent years. For the most part, this work has concentrated on the problem of allowing users of different proof assistants to share one another's work. This is a different problem to the one we consider in this paper: how to formalise a piece of mathematics that involves several different foundations. Nevertheless, the two lines of research should be able to benefit from one another.

6.1.1. Logosphere The large project Logosphere, started by Schürmann, Pfenning, Kohlhase and Owre, aims to build a large library of formalised proofs that users of many different proof assistants can all contribute to and make use of. It does this by representing the many different many different systems and the translations between them in the Edinburgh LF. In particular, Howe's translation from HOL to NuPRL (Howe, 1996; Howe, 1998) has been formalised and verified to be correct using the proof assistant Twelf (Schürmann and Stehr, 2006).

The representation of HOL in ELF consists of a type tp:type to represent the types of HOL, together with a function $\mathrm{tm}: \mathrm{tp} \rightarrow$ type to represent the terms of each type. The representation of Nuprl consists of a type $\mathrm{n}-\mathrm{tm}$ :type to represent the terms of Nuprl. The translation $\Phi$ is then represented by two functions

$$
\text { transtp:tp } \rightarrow \mathrm{n}-\mathrm{tm} \rightarrow \text { type } \quad \text { transtm:tm } A \rightarrow \mathrm{n}-\mathrm{tm} \rightarrow \text { type }
$$

with transtp $A B$ being inhabited iff $\Phi(A)=B$. Twelf is able to verify that transtp and transtm are total functions that satisfy the desired properties.

Their approach thus intended to be used to translate between two systems implemented in two different proof checkers, using a logical framework implemented in a third. Our approach is intended to be used by someone using just one proof checker, that implements a logical framework.

The most important difference is that they represent the translation as a pair of objects in the logical framework, and formally verifying its correctness. We do not represent the translation as an object; rather, it is effected by replacing a set of declarations with a set of definitions.

It remains to be seen how the two approaches compare in practice. However, we anticipate that our approach should be more convenient for a pluralist formalisation, as there is less overhead for the user. The translations are invisible to the user. Given a translation $\Phi: S \rightarrow T$, when the user is working in $T$, there is no need for them to invoke $\Phi$ in order to make use of the results proved in $S$. A change in the proof scripts produced in $S$ will produce an immediate change in the theorems that are visible to the user and available for use in $T$. 
6.1.2. Little Theories The proof assistant IMPS is designed to use the little theories methodology (Farmer, 2000), whereby a number of different theories and translations between them are specified using a version of higher-order logic called LUTINS (Farmer et al., 1990). A translation from one theory $T_{1}$ to another $T_{2}$ is specified by a mapping from the constants of $T_{1}$ to the expressions of $T_{2}$ satisfying certain conditions, such that the translation of every axiom of $T_{1}$ is a theorem of $T_{2}$.

The LTTs we have been dealing with in this paper have a richer type structure than the theories that can be specified in LUTINS. Our LTTs include dependent types, inductive types and computation rules. Apart from this difference, the class of translations that can be handled by the two methods is remarkably similar.

However, IMPS required support for translations to be built into the implementation. We have shown in this paper that, when working with a logical framework, a lightweight mechanism for supporting these translations is automatically available for free.

The work that has been done in IMPS shows how useful it can be to work with a variety of theories and translations in the course of a formalisation. We hope that the programme of research proposed in this paper will prove that this remains true when we are working in LTTs, too.

6.1.3. Other Methods of Proof Reuse The method of proof reuse between different systems of logic presented in this paper is, to the best of the authors' knowledge, original.

Previous work on proof reuse in dependent type theories has concentrated on proof reuse within a single type theory, in the following two specific classes of situations:

1 Given an isomorphism between types $A$ and $B$, to automatically generate proofs about $B$ from proofs about $A$ (Beckert and Klebanov, 2004).

2 Given an inductive type $A$, and an inductive type $B$ formed by extending $A$ with new constructors, to reuse proofs about $A$ to interactively generate proofs about $B$ (Boite, 2004).

3 The project LATIN (Iancu and Rabe, 2010) has similar aims to Logosphere.

4 Garillot and Gonthier's method of mathematical components (François Garillot et al., 2009 ) is a disciplined way of systematically organising the development of large formalisations involving many algebraic structures, in such a way that (e.g.) results about groups can be applied to rings. This is not the same problem as the one considered in this paper, which involves translations between systems that involve changing both the type structure and the logic. Nevertheless, there are superficial similarities, and it remains to be seen if the two methods have anything to offer each other.

\subsection{Future Work}

Plastic at present has a very primitive module mechanism, which nevertheless was sufficient for the work in this paper. However, the method we have presented relies on editing proof scripts, changing the files which they import, which is very inconvenient in practice.

A more sophisticated module system that allows for parameterised modules would make this form of proof reuse much more convenient. For our future work, we would like to implement such a module mechanism in Plastic. 
We would also like to establish a better theoretical basis for this type of work. We would like to establish some criteria for when a translation can be represented by a module by our method. We would like to study the theory of modules, module interfaces and module functors described above. The result will likely be very similar to the theory of institutions (Goguen and Burstall, 1984); there are many superficial similarities. Institutions are not exactly what we need, however, as institutions are a model-theoretic notion, whereas we require a syntactic notion.

\section{Acknowledgements}

We would like to thank the anonymous referees for their very helpful and detailed comments, and particularly for making us aware of related work in the area of proof reuse.

\section{References}

Aczel, P. (2001). The Russell-Prawitz modality. Mathematical Structures in Computer Science, 11(4):541-554.

Aczel, P. and Gambino, N. (2002). Collection principles in dependent type theory. In Luo, Z., McKinna, J., and Pollack, R., editors, Types for Proofs and Programs, volume 2277 of LNCS, pages 1-23. Springer.

Adams, R. and Luo, Z. (2007). Weyl's predicative classical mathematics as a logic-enriched type theory. In Altenkirch, T. and McBride, C., editors, Types for Proofs and Programs, volume 4502 of $L N C S$, pages $1-17$. Springer.

Adams, R. and Luo, Z. (2010). Weyl's predicative classical mathematics as a logic-enriched type theory. ACM Trans. on Computational Logic, 11(2):1-29.

Agda (2008). The Agda proof assistant. Available from the web page: http://appserv.cs.chalmers.se/users/ulfn/wiki/agda.php.

Beckert, B. and Klebanov, V. (2004). Proof reuse for deductive program verification. In SEFM '04 Proceedings of the Software Engineering and Formal Methods, Second International Conference, pages 77-86. Published by the IEEE Computer Society.

Bertot, Y. and Castéran, P. (2004). Interactive theorem proving and program development: Coq'Art: the calculus of inductive constructions. Springer-Verlag New York Inc.

Boite, O. (2004). Proof reuse with extended inductive types. In Slind, K., Bunker, A., and Gopalakrishnan, G., editors, Theorem Proving in Higher Order Logics, volume 3223 of LNCS, pages 50-65. Springer.

Callaghan, P. C. and Luo, Z. (2001). An implementation of typed LF with coercive subtyping and universes. J. of Automated Reasoning, 27(1):3-27.

Constable, R. et al. (1986). Implementing Mathematics with the NuPRL Proof Development System. Pretice-Hall.

Coq (2004). The Coq Proof Assistant Reference Manual (Version 8.0), INRIA. The Coq Development Team.

Coquand, T. and Huet, G. (1988). The calculus of constructions. Information and Computation, 76(2-3):95-120.

Coquard, T. and Paulin-Mohring, C. (1990). Inductively defined types. In Martin-Löf, P. and Mints, G., editors, COLOG-88: International Conference in Computer Logic, volume 417 of LNCS, pages 50-66. Springer-Verlag. 
Dybjer, P. (1991). Inductive sets and families in Martin-Löf's type theory and their set-theoretic semantics. In Huet, G. and Plotkin, G., editors, Logical Frameworks, pages 280-306. Cambridge University Press.

Farmer, W., Guttman, J., and Thayer, F. (1990). IMPS : An interactive mathematical proof system. In Stickel, M., editor, 10th International Conference on Automated Deduction, volume 449 of $L N C S$, pages 653-654. Springer.

Farmer, W. M. (2000). An infrastructure for intertheory reasoning. In McAllester, D., editor, Automated Deduction - CADE-17, volume 1831 of LNCS, pages 115-131.

Feferman, S. (2005). Predicativity. In Shapiro, S., editor, The Oxford Handbook of Philosophy of Mathematics and Logic. Oxford Univ Press.

François Garillot, Georges Gonthier, Assia Mahboubi, and Laurence Rideau (2009). Packaging Mathematical Structures. In Tobias Nipkow and Christian Urban, editors, Theorem Proving in Higher Order Logics, volume 5674 of Lecture Notes in Computer Science, Munich Allemagne. Springer.

Friedman, H. (1978). Classically and intuitionistically provable functions. In Higher Set Theory, pages $21-28$.

Gambino, N. and Aczel, P. (2006). The generalised type-theoretic interpretation of constructive set theory. J. of Symbolic Logic, 71(1):67-103.

Girard, J.-Y. (1986). The system F of variable types, fifteen years later. Theoretical Computer Science, 45(2):159-192.

Girard, J.-Y., Lafont, Y., and Taylor, P. (1990). Proofs and Types. Cambridge University Press.

Gödel, K. (1933). On intuitionistic arithmetic and number theory. Collected Works, pages $287-295$.

Goguen, H. (1994). A Typed Operational Semantics for Type Theory. PhD thesis, University of Edinburgh.

Goguen, J. and Burstall, R. (1984). Introducing institutions. Logics of Programs, pages 221-256.

Hájek, P. and Pudlák, P. (1998). Metamathematics of First-Order Arithmetic, volume 3 of Perspectives in Mathematical Logic. Springer.

Harper, R., Honsell, F., and Plotkin, G. (1987). A framework for defining logics. Proc. 2nd Ann. Symp. on Logic in Computer Science. IEEE.

Harper, R., Honsell, F., and Plotkin, G. (1993). A framework for defining logics. Journal of the Association for Computing Machinery, 40(1):143-184.

Harrison, J. (1996). HOL Light: A tutorial introduction. In Formal Methods in Computer-Aided Design, pages 265-269. Springer.

Howe, D. J. (1996). Importing mathematics from HOL into Nuprl. In von Wright, J., Grundy, J., and Harrison, J., editors, Theorem Proving in Higher Order Logics, 9th International Conference, TPHOLs'96, Turku, Finland, August 26-30, 1996, Proceedings, volume 1125 of $L N C S$, pages 267-282. Springer-Verlag.

Howe, D. J. (1998). Toward sharing libraries of mathematics between theorem provers. In Frontiers of Combining Systems, FroCoS'98, ILLC, University of Amsterdam, October 2-4, 1998, Proceedings. Kluwer Academic Publishers.

Iancu, M. and Rabe, F. (2010). Formalizing foundations of mathematics. Mathematical Structures in Computer Science.

Kohlenbach, U. (2005). Higher order reverse mathematics. In Simpson, S., editor, Reverse mathematics 2001, volume 21 of Lecture Notes in Logic, pages 281-295. Assoc. Symbol. Logic and A. K. Peters.

Luo, Z. (1994). Computation and Reasoning: A Type Theory for Computer Science. Oxford University Press. 
Luo, Z. (2003). $\mathrm{PAL}^{+}$: a lambda-free logical framework. Journal of Functional Programming, $13(2): 317-338$.

Luo, Z. (2006). A type-theoretic framework for formal reasoning with different logical foundations. In Okada, M. and Satoh, I., editors, Proc of the 11th Annual Asian Computing Science Conference, volume 4435 of LNCS. Tokyo.

Luo, Z. and Pollack, R. (1992). LEGO Proof Development System: User's Manual. LFCS Report ECS-LFCS-92-211, Department of Computer Science, University of Edinburgh.

Martin-Löf, P. (1984). Intuitionistic Type Theory. Bibliopolis.

Muzalewski, M. (1993). An Outline of PC Mizar. Fondation Philippe le Hodey, Brussels.

Nipkow, T., Paulson, L. C., and Wenzel, M. (2002). Isabelle/HOL: a proof assistant for higherorder logic. LNCS Tutorial 2283.

Nordström, B., Petersson, K., and Smith, J. (1990). Programming in Martin-Löf's Type Theory: An Introduction. Oxford University Press.

Paulson, L. C. (1994). Isabelle: a generic theorem prover. Lecture Notes in Computer Science, 828.

Pfenning, F. and Schürmann, C. (1999). Twelf - a meta-logical framework for deductive systems. In Ganzinger, H., editor, Automated Deduction - CADE-16, volume 1632 of LNCS, pages 202-206. Springer.

Pollack, R. (1994). The Theory of LEGO: A Proof Checker for the Extended Calculus of Constructions. PhD thesis, Edinburgh University.

Russell, B. (1903). The Principles of Mathematics. Routledge.

Schürmann, C. and Stehr, M.-O. (2006). An executable formalization of the HOL/Nuprl connection in the metalogical framework Twelf. In Hermann, M. and Voronkov, A., editors, Proceedings of Logic for Programming, Artificial Intelligence and Reasoning (LPAR), volume 4246 of Lecture Notes in Artificial Intelligence, pages 150-166. Springer-Verlag.

Schütte, K. (1965). Predicative well-orderings. Studies in Logic and the Foundations of Mathematics, 40:280-303.

Shoenfield, J. (1967). Mathematical logic. Addison-Wesley Reading, Mass.

Simpson, S. G. (1999). Subsystems of Second-Order Arithmetic. Springer-Verlag.

Smith, J. (1988). The independence of Peano's fourth axiom from Martin-Löf's type theory without universes. Journal of Symbolic Logic, 53(3).

Weyl, H. ((English translation of Das Kontinuum, 1918)). The Continuum: a critical examination of the foundation of analysis. Dover Publ. 1994. 